\title{
Candidate CSPG4 mutations and induced pluripotent stem cell modeling implicate oligodendrocyte progenitor cell dysfunction in familial schizophrenia
}

\author{
Femke M. de Vrij ${ }^{1}$ Christian G. Bouwkamp ${ }^{1,2}$ - Nilhan Gunhanlar ${ }^{1} \cdot$ Guy Shpak $^{1}$ - Bas Lendemeijer ${ }^{1}$. \\ Maarouf Baghdadi ${ }^{1}$ - Shreekara Gopalakrishna ${ }^{1}{ }^{1} \cdot$ Mehrnaz Ghazvini ${ }^{3} \cdot$ Tracy M. $\mathrm{Li}^{3} \cdot$ Marialuisa Quadri $^{2}$. \\ Simone Olgiati ${ }^{2} \cdot$ Guido J. Breedveld ${ }^{2} \cdot$ Michiel Coesmans ${ }^{1,4} \cdot$ Edwin Mientjes $^{5,6} \cdot$ Ton de Wit $^{2} \cdot$ Frans W. Verheijen $^{2}$. \\ H. Berna Beverloo ${ }^{2}$ Dan Cohen ${ }^{7} \cdot$ Rob M. Kok ${ }^{8}$ P. Roberto Bakker ${ }^{9,10} \cdot$ Aviva Nijburg $^{8} \cdot$ Annet T. Spijker $^{8}$. \\ P.M. Judith Haffmans ${ }^{11}$ - Erik Hoencamp ${ }^{8,12}$ - Veerle Bergink ${ }^{1}$. GROUP Study Consortium • \\ Jacob A. Vorstman ${ }^{13,14,15} \cdot$ Timothy $\mathrm{Wu}^{16} \cdot$ Loes M. Olde Loohuis $\mathbb{1}^{16} \cdot$ Najaf Amin ${ }^{17}$. Carolyn D. Langen ${ }^{18,19,20}$. \\ Albert Hofman ${ }^{17} \cdot$ Witte J. Hoogendijk ${ }^{1}$ Cornelia M. van Duijn ${ }^{17} \cdot$ M. Arfan Ikram $\mathbb{1}^{17,18,21} \cdot$ Meike W. Vernooij ${ }^{17,18}$. \\ Henning Tiemeier $\mathbb{D}^{1,17,19} \cdot$ André G. Uitterlinden ${ }^{17,22} \cdot$ Ype Elgersma $\mathbb{D}^{5,6} \cdot$ Ben Distel $^{23} \cdot$ Joost Gribnau $^{3}$. \\ Tonya White $^{19} \cdot$ Vincenzo Bonifati $^{2} \cdot$ Steven A. Kushner ${ }^{1}{ }^{1}$
}

Received: 11 November 2016 / Revised: 24 September 2017 / Accepted: 1 November 2017 / Published online: 4 January 2018

(c) The Author(s) 2017. This article is published with open access

\begin{abstract}
Schizophrenia is highly heritable, yet its underlying pathophysiology remains largely unknown. Among the most well-replicated findings in neurobiological studies of schizophrenia are deficits in myelination and white matter integrity; however, direct etiological genetic and cellular evidence has thus far been lacking. Here, we implement a family-based approach for genetic discovery in schizophrenia combined with functional analysis using induced pluripotent stem cells (iPSCs). We observed familial segregation of two rare missense mutations in Chondroitin Sulfate Proteoglycan 4 (CSPG4) (c.391G > A [p.A131T], MAF $7.79 \times 10^{-5}$ and c. $2702 \mathrm{~T}>\mathrm{G}[\mathrm{p} . \mathrm{V} 901 \mathrm{G}]$, MAF $2.51 \times 10^{-3}$ ). The CSPG4 $4^{A l 31 T}$ mutation was absent from the Swedish Schizophrenia Exome Sequencing Study (2536 cases, 2543 controls), while the CSPG $4^{V 901 G}$ mutation was nominally enriched in cases (11 cases vs. 3 controls, $P=0.026$, OR $3.77,95 \%$ CI 1.05-13.52). CSPG4/NG2 is a hallmark protein of oligodendrocyte progenitor cells (OPCs). iPSC-derived OPCs from CSPG4 ${ }^{A 13 l T}$ mutation carriers exhibited abnormal posttranslational processing $(P=0.029)$, subcellular localization of mutant NG2 $(P=0.007)$, as well as aberrant cellular morphology $\left(P=3.0 \times 10^{-8}\right)$, viability $\left(P=8.9 \times 10^{-7}\right)$, and myelination potential $(P=0.038)$. Moreover, transfection of healthy noncarrier sibling OPCs confirmed a pathogenic effect on cell survival of both the CSPG4 ${ }^{A l 3 l T}(P=0.006)$ and $C S P G 4^{V 901 G}$ $\left(P=3.4 \times 10^{-4}\right)$ mutations. Finally, in vivo diffusion tensor imaging of $C S P G 4^{A 131 T}$ mutation carriers demonstrated a reduction of brain white matter integrity compared to unaffected sibling and matched general population controls $\left(P=2.2 \times 10^{-5}\right)$. Together, our findings provide a convergence of genetic and functional evidence to implicate OPC dysfunction as a candidate pathophysiological mechanism of familial schizophrenia.
\end{abstract}

Investigators from the GROUP Study Consortium are listed in the Supplement.

Femke M. de Vrij, Christian G. Bouwkamp, and Nilhan Gunhanlar contributed equally to this work.

Electronic supplementary material The online version of this article (https://doi.org/10.1038/s41380-017-0004-2) contains supplementary material, which is available to authorized users.

Steven A. Kushner

s.kushner@erasmusmc.nl

Extended author information available on the last page of the article

\section{Introduction}

Schizophrenia is a severely debilitating psychiatric disorder affecting $\sim 1 \%$ of the population worldwide [1]. The strongest known determinant for developing schizophrenia is family history. A meta-analysis, which included five decades of twin studies, concluded a heritability estimate $\left(h^{2}\right)$ of $0.77 \pm 0.05$, with a relatively limited contribution of shared environmental influences $\left(c^{2}\right)$ $(0.013 \pm 0.025)[2]$. 
The Psychiatric Genomics Consortium recently reported a genome-wide association study (GWAS) investigating 36,989 cases and 113,075 controls, in which 128 genomewide significant single-nucleotide variants were identified across 108 independent genomic loci, suggesting an important contribution of common genetic variation to schizophrenia risk [3]. However, a large proportion of the heritability for schizophrenia remains unexplained, leaving many genetic variants remaining to be discovered. Therefore, increasing attention has also been focused on the potential contribution of rare genomic variation to schizophrenia risk. Copy number variants (CNVs) are a well-established source of pleiotropic risk, ranging from asymptomatic carriership to a complex constellation of symptoms affecting multiple organ systems, such as the $22 \mathrm{q} 11.2$ microdeletion syndrome [4]. In a large-scale schizophrenia case-control cohort analysis, known pathogenic CNVs were significantly more frequent in cases $(2 \%)$ than controls $(0.4 \%)$ [5, 6]. Moreover, an independent study found that large $(>500 \mathrm{~kb}) \mathrm{CNVs}$ are enriched in loci associated with schizophrenia by GWAS and frequently involve genes encoding proteins located in the postsynaptic density [7].

In addition to microarray-based genotyping methods, the development of next-generation sequencing has allowed the possibility to examine whether rare single nucleotide variants or small insertions-deletions contribute to schizophrenia risk. A Swedish cohort including 2536 cases and 2543 controls yielded no single mutation or single gene reaching genome-wide significance for association with schizophrenia, but confirmed a similar enrichment of gene sets for synaptic function as previously identified for genes located in schizophrenia-associated CNVs and GWAS loci [8]. Moreover, trio-based studies have identified a number of candidate genes through identification of recurrent de novo mutations $[9,10]$ and an increased burden of mutations occurring in genes encoding glutamatergic postsynaptic proteins [11].

Recent genetic and induced pluripotent stem cell (iPSC)based studies have converged on a model by which neuronal function, and in particular synaptic transmission, is a major pathophysiological mechanism of schizophrenia [3, 8, 11-13]. However, functional neuronal alterations may arise either by direct cell-type autonomous changes to neurons themselves, or indirectly through a primary pathophysiological influence on other cell types that influence neuronal function. Numerous studies have reported the involvement of glial cell biology in the pathophysiology of schizophrenia, including alterations in oligodendrocytes, myelination, and white matter integrity [14-19], which directly regulate neuronal function.

Abnormalities of the integrity of the white matter are strongly associated with schizophrenia [20]. The late adolescent critical period for cerebral cortex myelination has long been recognized as overlapping closely with the typical age of onset for schizophrenia [16, 17]. Myelination-related genes have been shown to be enriched for common variants associated independently to white matter integrity [21] and schizophrenia [15, 22]. Two recent brain imaging studies have elegantly compared white matter integrity in $16 \mathrm{p} 11.2$ deletion and duplication carriers [23, 24] of which only the $16 \mathrm{p} 11.2$ duplication confers increased risk for schizophrenia [6]. Notably, both global fractional anisotropy (FA) and white matter volume were selectively decreased in $16 \mathrm{p} 11.2$ duplication carriers. However, despite increasing evidence of an association between schizophrenia and myelination integrity, the molecular and cellular mechanisms by which oligodendrocyte lineage dysfunction might influence schizophrenia risk have remained largely unknown.

We now report genetic and functional evidence of oligodendrocyte progenitor cell dysfunction in schizophrenia. Using a family-based genetic approach, we observed multiple rare missense mutations in CSPG4 that segregate with schizophrenia. Functional studies using iPSCs reprogrammed from affected CSPG4 mutation carriers and their unaffected non-carrier siblings revealed that patient-derived OPCs exhibit abnormal post-translational processing, aberrant subcellular localization of CSPG4/NG2, abnormal cellular morphology, reduced cellular viability, and impaired oligodendrogenesis. Moreover, diffusion tensor imaging (DTI) of CSPG4 mutation carriers confirmed a global impairment in white matter integrity, together providing support for OPC dysfunction as a candidate pathophysiological mechanism of schizophrenia.

\section{Methods summary}

\section{Genetic analysis}

Linkage and copy number analysis was performed with Illumina HumanCytoSNP-12v2 chip arrays using an affected-only model with an assumption of $99.9 \%$ penetrance. Analysis revealed a total of $294.34 \mathrm{Mb}$ of genomic regions, with suggestive linkage on chromosomes $2,11,14$, 15 , and 16. Whole-genome exome sequencing was performed twice: initially at $40 \times$, and again at $90 \times$ coverage. Exome variants were considered for additional validation if they were rare (minor allele frequency $(\mathrm{MAF})<0.001$ ), predicted to alter coding sequence (missense, nonsense, frameshift, essential splice site), and occurred within the regions of suggestive linkage.

\section{Cellular studies}

Human iPSCs were differentiated to neural progenitor cells (NPCs) and neurons by embryoid body-based neural 
differentiation [25]. Electrophysiology was performed in whole-cell patch-clamp configuration after 8-10 weeks of differentiation. iPSC-derived OPCs were differentiated according to Monaco et al. [26] with modifications. Biotinylation of cell surface proteins was adapted from Huang et al. [27]. OPC viability was assessed by quantitative fluorometric monitoring of resazurin conversion to resorufin [28]. Myelination assay was performed using ex vivo organotypic cerebral cortex slices of Shiverer mice, as previously described $[29,30]$.

\section{Magnetic resonance imaging (MRI)}

Two patients and one control sibling of the discovery family were subjected to MRI scanning. Population controls $(n=$ 294) matched on age, gender, and pack-years of cigarette smoking were selected from the Rotterdam Study. MRI images were obtained using a 1.5 Tesla General Electric (GE Healthcare, Milwaukee, Wisconsin, USA) MR system using a bilateral phased-array head coil. A full description of the imaging protocol and Rotterdam Study design has been described elsewhere [31]. An in-house MATLAB (Mathworks, Natick, MA) program was used to quantify the number and spatial characteristics of white matter "potholes" along the major white matter tracts [32].

\section{Results}

\section{Genetic findings in the discovery family}

A non-consanguineous family of Dutch ancestry was ascertained with a pattern of schizophrenia inheritance compatible with autosomal dominant transmission. The core pedigree consisted of a couple and their nine children (five males, four females) of whom the father and four sons suffered from non-syndromic schizophrenia (Fig. 1a and Supplementary Table 1).

Genome-wide parametric linkage analysis was performed on peripheral blood DNA using an autosomaldominant, affected-only model of inheritance, in order to identify genomic regions shared among all affected family members (Supplementary Table 2). Whole exome sequencing was performed on three individuals of the family (pedigree IDs: II-2, III-5, and III-9; Fig. 1a). Five candidate heterozygous variants were identified based on the following criteria: (a) located within the genomic regions shared among all affected family members, (b) predicted to affect protein coding (missense, nonsense, frameshift, splice site), (c) called in at least one of the affected individuals (III-5 and III-9), (d) absent from the unaffected mother (II-2), (e) absent from dbSNP129, and (f) with a MAF $<0.001$ in the Exome Aggregation Consortium (ExAC) browser
(Europeans non-Finnish) [33], EVS6500 European Americans, NHLBI Exome Sequencing Project (ESP) [34], 1000 Genomes [35], and Genome of the Netherlands (GoNL) [36] cohorts (Supplementary Table 3). Genotyping of these variants was performed by Sanger sequencing in all participating family members.

Among the five candidate variants, CSPG4 c.391G > A (p.A131T) was the only variant shared by all affected family members and absent in all unaffected relatives, including in the extended family (Fig. 1a). CSPG4 c.391G $>$ A (p.A131T) is present in the Exome Aggregation Consortium Browser (Total [forward strand]: $\mathrm{T}=6 / \mathrm{C}=$ 118,148 alleles [MAF $5.08 \times 10^{-5}$ ], European (Non-Finnish): $\mathrm{T}=5 / \mathrm{C}=64,215$ alleles [MAF $7.79 \times 10^{-5}$ ]) [33] but absent from the Swedish Schizophrenia Exome Sequencing Study (2536 cases, 2543 controls) [8], 1000 Genomes [35], and GoNL [36].

\section{Additional genotyping of CSPG4 discovery family variant}

In an effort to further characterize the frequency of CSPG4 c.391G > A (p.A131T) in the Netherlands, we performed TaqMan genotyping and Sanger sequencing validation in an independent Dutch cohort of 1219 schizophrenia cases and in the general population-based Rotterdam Study cohort [37] (10,611 subjects). One carrier was identified among the schizophrenia cases $\left(\mathrm{MAF}_{\text {cases }} 4.1 \times 10^{-4}\right)$ and three within the general population $\left(\mathrm{MAF}_{\text {population }} 1.4 \times 10^{-4}\right)$. The patient carrier had a long history of severe psychiatric illness, including multiple hospital admissions and chronic antipsychotic and antidepressant medication. Her most recent prescriptions included penfluridol oral depot $(20 \mathrm{mg} / \mathrm{week})$ and venlafaxine $(37.5 \mathrm{mg} /$ day $)$. Family members could not be ascertained for additional psychiatric history or genotyping. Among the three unrelated carriers (maximum pairwise $\hat{\pi}=0.078$ ) identified in the Rotterdam Study general population cohort, two had a clinically significant history of psychiatric illness $(\hat{\pi}=0.055)$. One of the subjects had a history of multiple inpatient psychiatric hospitalizations for depression, and the other required chronic antidepressant and anxiolytic pharmacotherapy. Notably these findings were unlikely due to chance alone, given the $12.76 \%$ period prevalence of antidepressant use in the Rotterdam Study cohort and $0.065 \%$ annual prevalence of inpatient psychiatric hospitalization in the Netherlands [38, 39] (Binomial $P=8.8 \times 10^{-3}$ ).

\section{Identification of a second rare CSPG4 variant that segregates with schizophrenia}

A previous study identified suggestive linkage at chromosome 15q22-24, containing CSPG4, in a cohort of 
Fig. 1 Familial segregation of CSPG4 mutations with schizophrenia. a Pedigree of multiplex discovery family with schizophrenia. Symbols: filled, schizophrenia; open, unaffected; G/A, heterozygous carrier of the CSPG4 c.391G > A mutation $\left(C S P G 4^{A 131 T}\right) ; \mathrm{G} / \mathrm{G}$,

homozygous reference. b

Representative sequencing results for heterozygous carriers of the CSPG4 c. $391 \mathrm{G}>\mathrm{A}$ mutation. The lower panel reflects homozygous reference sequence. c Homology model of the first Laminin $G$ domain of CSPG4. Structural alignment of the reference (green) and mutant model (magenta) reveals a difference in the predicted interaction between amino acid positions 131 and 96 (Leu) in the opposing $\beta$-strand inside the hydrophobic core of the $\beta$ sandwich (predicted alterations of the side chains in red). $\mathbf{d}$ Family pedigrees segregating the $C S P G 4$ c. $2702 \mathrm{~T}>\mathrm{G}$ mutation $\left(C S P G 4^{V 901 G}\right)$. Symbols: filled, schizophrenia; open, unaffected; T/G,

heterozygous carrier of the CSPG4 c.2702T > G mutation; $\mathrm{T} / \mathrm{T}$, homozygous reference. $\mathbf{e}$ Three-dimensional structural homology modeling of the putative 3rd LamG domain of CSPG4 (a.a. 634-921), demonstrating the outside surface location of $\mathrm{Val}^{901}$. $\mathbf{f}$ Representative Sanger sequencing trace of heterozygous carrier of the CSPG4 c. $2702 \mathrm{~T}>\mathrm{G}$ mutation a
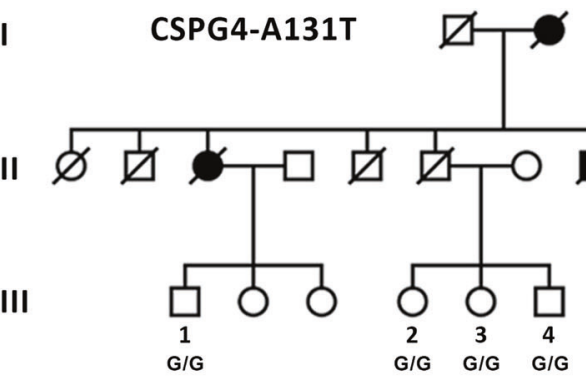

b
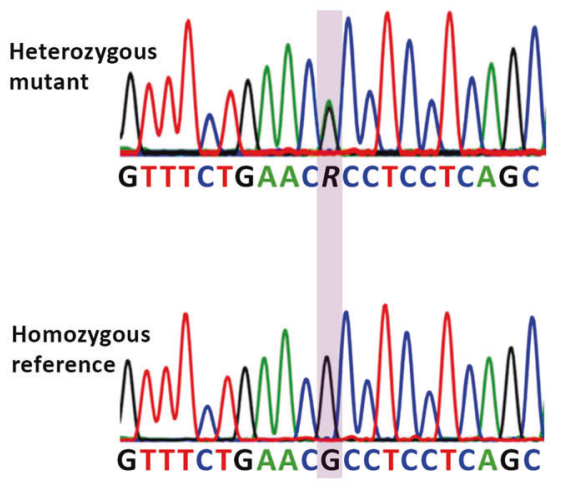

d

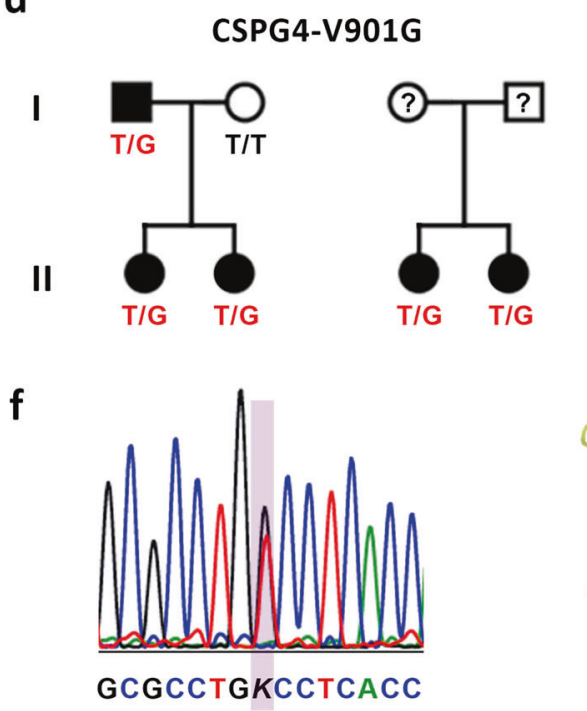

C
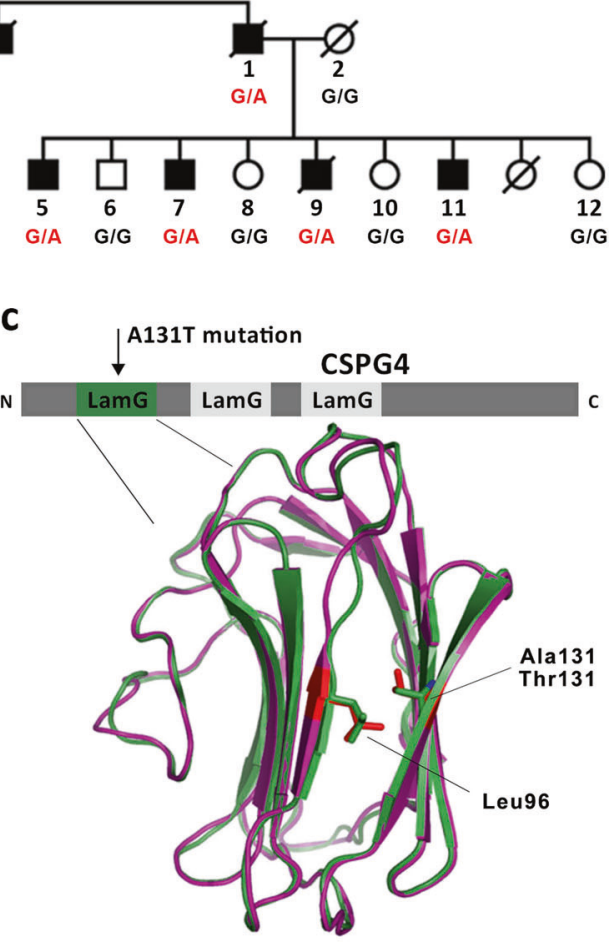

e
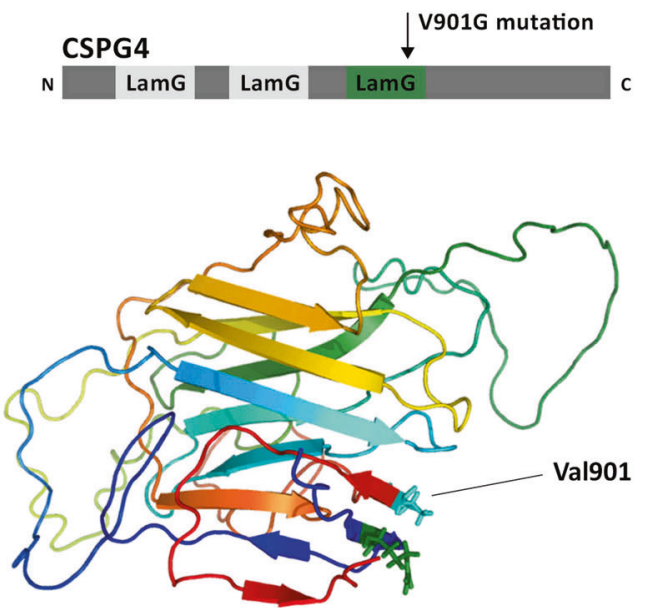

175 families with schizophrenia or schizoaffective disorder of Central American/Hispanic origin [40, 41]. We therefore sequenced the full open reading frame of CSPG4 in one proband from each of the 73 families that positively contributed to the linkage signal at this locus (markers D15S131 and D15S655) (Supplementary Table 4). Four rare missense variants were identified with $\mathrm{MAF}<0.005$ (ExAC Browser Latino) (Fig. 1, Supplementary Fig. 1). However in contrast to the other three variants, CSPG4
c.2702T $>$ G (p.V901G) was found in two independent families, without evidence of incomplete penetrance (Fig. 1d, f), and located within a LamG domain similar to the c.391G $>$ A (p.A131T) discovery family variant (Fig. 1e). Moreover, the c. $2702 \mathrm{~T}>\mathrm{G}$ p.V901G variant was nominally enriched in cases from the Swedish Schizophrenia Exome Sequencing Study [8] $\left(\mathrm{MAF}_{\text {cases }} 2.19 \times 10^{-3} ; \mathrm{MAF}_{\text {controls }} 5.82 \times 10^{-4}\right.$, Fisher's Exact Test $P=0.026$, OR 3.77, 95\% CI 1.05-13.52). Together, these findings suggest that rare 
coding variants of CSPG4 might influence the risk of familial schizophrenia.

\section{Structural protein modeling of CSPG4 mutations}

The CSPG4 $4^{A 131 T}$ and CSPG4 $4^{V 901 G}$ mutations are located within the first and third Laminin $\mathrm{G}$ domain of the protein encoded by CSPG4, known as neural/glial 2 (NG2) (Fig. 1c, e). Laminin $\mathrm{G}$ (LamG) domains are highly conserved across a diverse group of extracellular matrix proteins [42]. Intriguingly, several schizophrenia-associated genes such as NRXN1 and LAMA2 also contain LamG domains [10, 4345]. Crystal structures of LamG domains in the Protein Data Bank allowed homology modeling of the LamG domains of NG2. Models implemented using Phyre2 [46] and I-TASSER [47] both suggested that in the reference sequence, $\mathrm{Ala}^{131}$ and $\mathrm{Leu}^{96}$ interact across opposing $\beta$-sheets inside the hydrophobic core of the $\beta$-sandwich. The mutation of $\mathrm{Ala}^{131}$, which has a small hydrophobic side chain, to $\mathrm{Thr}^{131}$, containing a larger polar side chain, suggests a conformational change impairing the proper folding of the $\beta$-sandwich (Fig. 1c).

Interestingly, $C S P G 4^{V 901 G}$ is located in a putative $3^{\text {rd }}$ LamG domain predicted by I-TASSER [47] (a.a. 634-921, Fig. 1e). This region has not previously been annotated as a LamG domain, despite the striking structural homology to other LamG domains with available crystal structures, most notably that of NRXN1 [48-50]. In contrast to the $C S P G 4^{A 131 T}$ mutation that is located on the inside of the globular structure of the first LamG domain of NG2, the $C S P G 4^{V 901 G}$ mutation is predicted to be located on the outside of the putative third LamG domain (Fig. 1e), therefore perhaps affecting protein-protein interactions. Intriguingly, the same protein region has been found to bind to collagen $\mathrm{V}$ and $\mathrm{VI}$, implicated in cell adhesion and migration of NG2-expressing cells [51, 52].

\section{Family-based iPSC modeling of the CSPG4 ${ }^{A 131 T}$ mutation}

\section{No evidence for a cell-autonomous neuronal phenotype}

Recent genetic and iPSC-based studies of schizophrenia have converged on a model by which neuronal function, and in particular synaptic transmission, is a major pathophysiological mechanism [3, 8, 11-13]. We obtained skin biopsies for iPSC reprogramming from three affected $C S P G 4^{A l 3 l T}$ carriers and three unaffected non-carriers within the core sibship of the discovery family (Supplementary Fig. 2a-d). Directed differentiation of iPSCs yielded forebrain-specified NPCs uniformly positive for Nestin, SOX2, Vimentin, and FOXG1 (Supplementary Fig. 2e).
NPCs were differentiated to neural cultures for 8-10 weeks, which notably lack cells of the oligodendrocyte lineage including OPCs. Both control and patientderived neurons developed robust synaptic network connectivity, confirmed by confocal immunofluorescence (Fig. 2a-c) and whole-cell patch-clamp electrophysiological recordings (Fig. 2d-r). Overall, neurons derived from patient carriers and their unaffected siblings had largely similar electrophysiological properties, including passive membrane properties, action potential characteristics, and synaptic physiology. However, two electrophysiological parameters were significantly different between patient and control iPSC-derived neurons-input resistance (control: $1233 \pm 88.9 \mathrm{~m} \Omega$, patient: $1605 \pm 112 \mathrm{~m} \Omega ; t_{50}=2.54, P=$ 0.01 ) and AP threshold (control: $-51.52 \pm 0.77 \mathrm{mV}$, patient: $\left.-48.63 \pm 0.67 \mathrm{mV} ; t_{49}=2.84, P=0.007\right)$.

\section{Abnormal post-translational processing and subcellular localization of CSPG4 ${ }^{A 131 T}$ in OPCs}

Given the highly abundant expression of NG2 in OPCs, widely referred to as NG2 cells, we next sought to investigate the influence of the CSPG4 $4^{A 131 T}$ mutation on iPSCderived OPCs. Directed differentiation of iPSCs to OPCs resulted in robust expression of the lineage-specific markers NG2, PDGFR $\alpha$, Olig2, and SOX10 (Supplementary Fig. 3). We first examined the subcellular distribution of NG2, as the structural homology modeling of the CSPG4 $4^{A 131 T}$ mutation suggested aberrant protein folding (Fig. 1c). Since NG2 is a transmembrane protein, it requires processing by the secretory pathway. Consistent with an impairment of protein processing, CSPG4 $4^{A 131 T}$ patient-derived OPCs showed a highly abnormal subcellular localization of NG2 exemplified by an increase of co-localization with the endoplasmic reticulum marker calreticulin $(t=5.08$, $P=0.007)$ (Fig. 3a, b).

In order to further characterize the alteration of NG2 subcellular localization, we performed surface biotinylation of $C S P G 4^{A 13 I T}$ patient and non-carrier sibling control OPCs. NG2 is known to undergo extensive posttranslational modification [53,54], including the addition of chondroitin sulfate moieties at $\operatorname{Ser}^{999}$ [55]. Consequently, NG2 appears as multiple bands by western blotting: a sharp band at $300 \mathrm{kDa}$ corresponding to an unmodified form of NG2 which lacks chondroitin sulfate side chains, and a large polydisperse smear at $>300 \mathrm{kDa}$ corresponding to NG2 with chondroitin sulfate modification. Pre-incubation with chondroitinase $\mathrm{ABC}$ to enzymatically cleave the chondroitin sulfate side chains eliminated the $>300 \mathrm{kDa}$ polydisperse smear (modified NG2) and increased the $300 \mathrm{kDa}$ band (unmodified NG2) (Supplementary Fig. 4a).

The total level of NG2 protein was similar between patient and control OPCs in whole-cell lysates $(t=0.51$, 

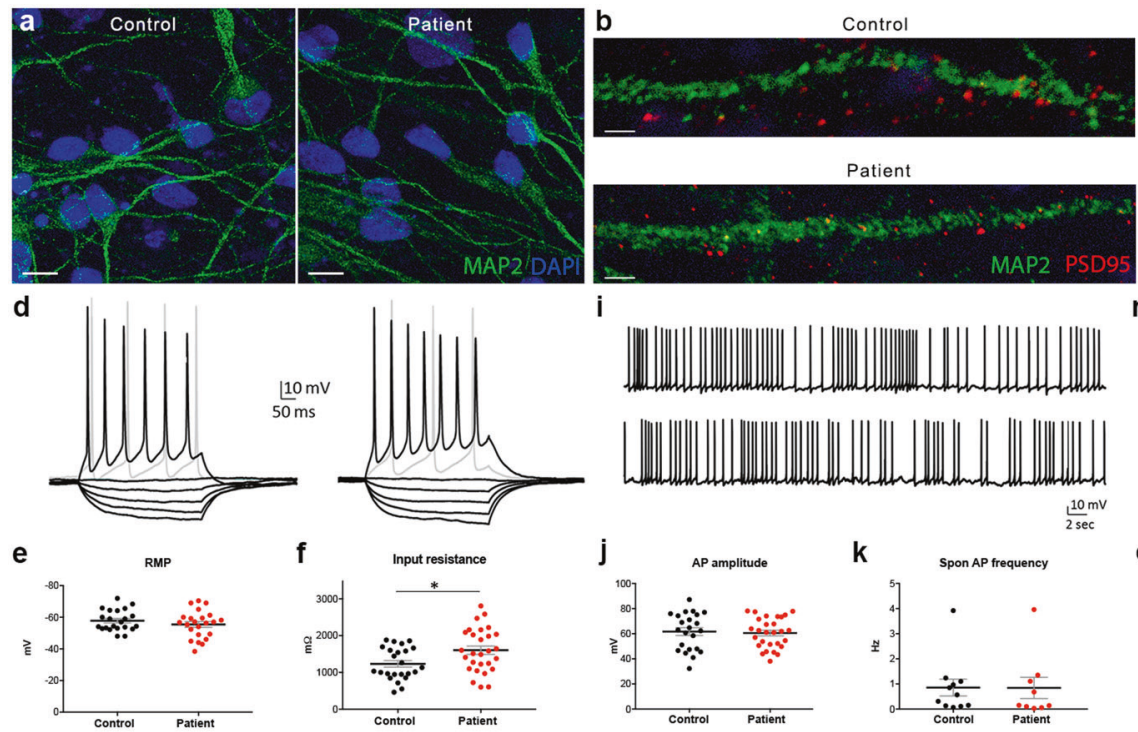

g

h
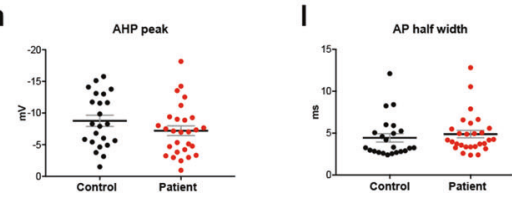

Fig. 2 Normal passive, active, and synaptic function in $C S P G 4^{A 131 T}$ patient iPSC-derived neurons. a Immunostaining of iPSC-derived neural cultures after 8 weeks of differentiation (scale bar $=10 \mu \mathrm{m})$. b, c Immunostaining with MAP2, PSD95, and synapsin antibodies confirmed the presence of synaptic proteins on dendrites of iPSC-derived neurons 8 weeks post differentiation (scale bar $=2 \mu \mathrm{m}$ ). d-r Electrophysiological measurements of iPSC-derived neurons. d Representative voltage responses to hyperpolarizing (range: $-20-0 \mathrm{pA}, 5 \mathrm{pA}$ increments) and depolarizing (10 and $20 \mathrm{pA})$ current steps (left: control, right: patient). e Resting membrane potential (RMP) $\left(t_{44}=1.04\right.$, $P=0.30)$. f Input resistance $\left(t_{50}=2.54, P=0.01\right)$. g Current-voltage $(\mathrm{I}-\mathrm{V})$ relationship of patient and control cells. $\mathbf{h}$ AHP peak $\left(t_{49}=1.35\right.$, $P=0.18$ ). i Representative traces of spontaneous action potential (AP)

$P=0.62$ ) (Supplementary Figs. 4, 5). However, patient OPCs exhibited a significant decrease in the ratio of modified vs. unmodified NG2 compared to control OPCs. This finding was observed in whole-cell lysate $(t=2.88$, $P=0.04)$, as well as independently in the intracellular $(t=3.50, P=0.02)$ and surface protein fractions of OPCs $(t=3.31, P=0.03$ ) (Supplementary Fig. 4b, c). Taken together, these results demonstrate that the CSPG4 $4^{A 131 T}$ mutation results in abnormal processing of NG2 protein.

\section{Abnormal morphology of OPCs derived from CSPG4 $4^{A 131 T}$ mutation carriers}

In addition to the abnormal processing of NG2 protein, we also observed distinct morphological differences between OPCs derived from patients and controls (Fig. 3c). Patientderived OPCs exhibited a size distribution that was strongly shifted toward smaller cells, a finding that was highly

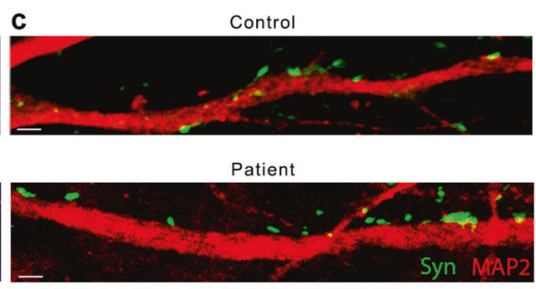

n

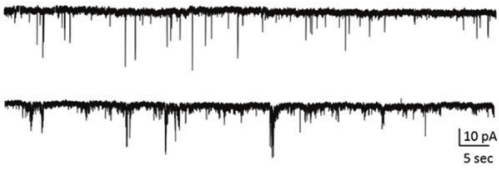

K

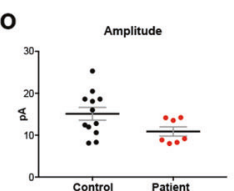

p

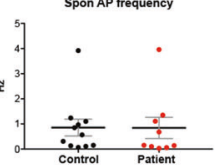

m
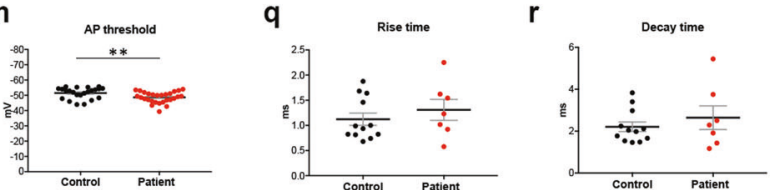

firing (50 s at RMP; top: control, bottom: patient). j AP amplitude ( $t_{49}$ $=0.31, P=0.76)$. $\mathbf{k}$ Spontaneous firing rate $\left(t_{19}=0.03, P=0.98\right)$. $\mathbf{~}$ AP half-width $\left(t_{49}=0.65, P=0.52\right)$. m AP voltage threshold $\left(t_{49}=2.84, P=0.007\right)$. n Representative traces of spontaneous postsynaptic currents $(100 \mathrm{~s}$ at $-90 \mathrm{mV}$; top: control, bottom: patient). o sPSC amplitude $\left(t_{17}=1.94, P=0.07\right)$. p sPSC frequency $\left(t_{17}=0.65\right.$, $P=0.52)$. q sPSC rise time $\left(t_{17}=0.84, P=0.41\right)$. $\mathbf{r}$ sPSC decay time $\left(t_{17}=0.84, P=0.41\right)$. d-r Unpaired two-tailed $t$-test. Passive properties and evoked APs $(N=24$ control, $N=28$ patient). Spontaneous APs $(N=11$ control, $N=9$ patient). Spontaneous postsynaptic currents $(N=12$ control, $N=7$ patient). All error bars are $+/-$ standard errors of the mean (SEM)

significant across all patient and control lines (Kolmogorov-Smirnov $D=0.25, P=3.0 \times 10^{-8}$; Fig. $3 \mathrm{~d}$ ). Overall, these results suggest that abnormal processing of NG2 influences the function of OPCs derived from CSPG $4^{A 131 T}$ mutation carriers.

\section{Patient OPCs have reduced viability and oligodendrogenesis}

Abnormally high co-localization of mutant NG2 with calreticulin is consistent with retention of misfolded mutant NG2 in the endoplasmic reticulum. The extensive literature demonstrating impairments of cell viability resulting from misfolded proteins $[56,57]$ led us to hypothesize that the abnormal subcellular distribution of mutant NG2 might reduce the viability of OPCs. Therefore, we performed a longitudinal monitoring of cell viability using a quantitative fluorescence-based indicator based on the conversion of 
a

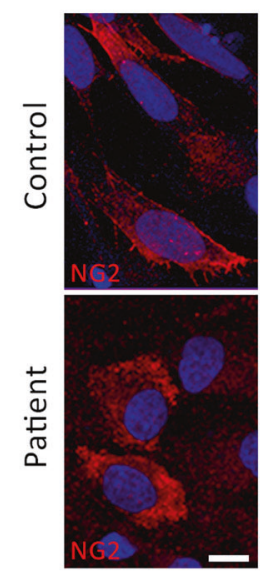

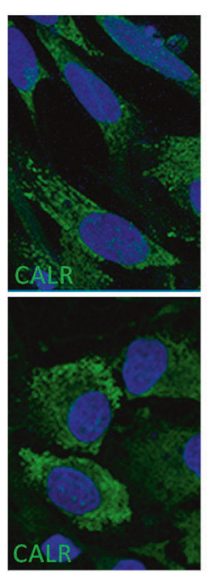

C

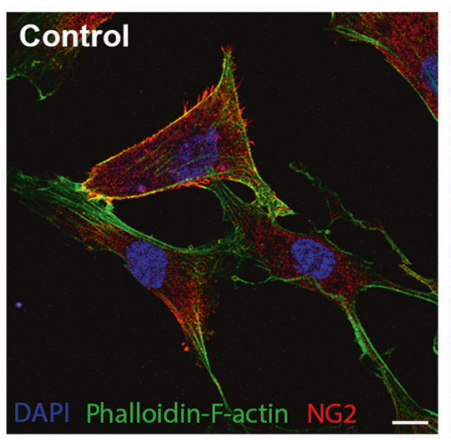

e

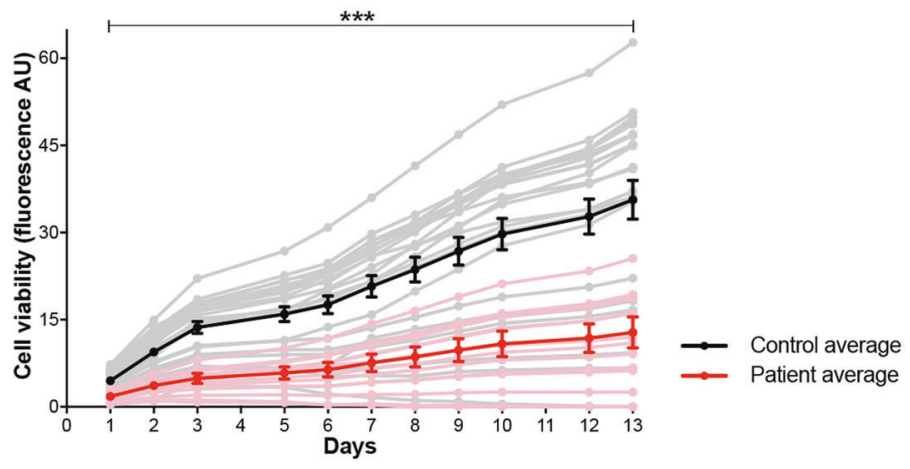

f

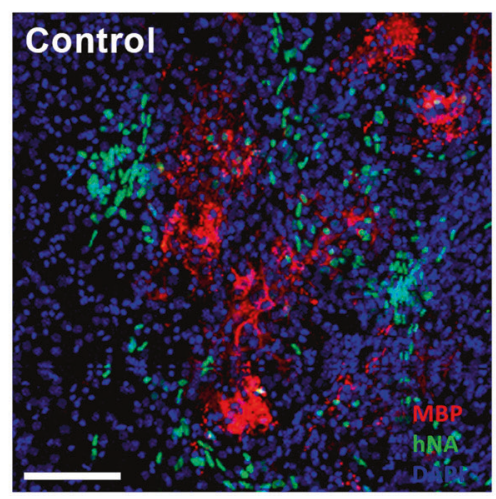

b

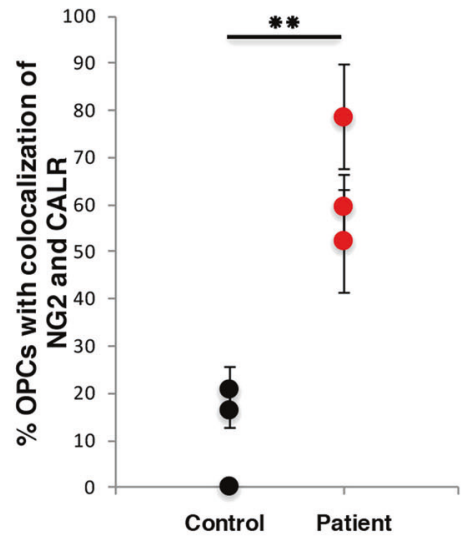

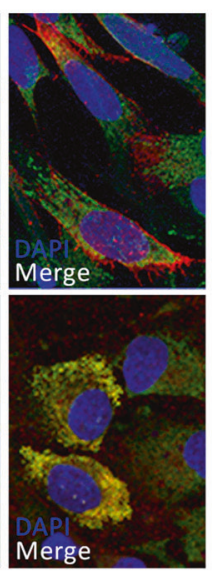

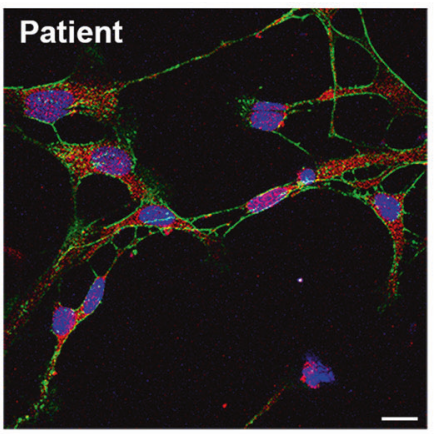

d

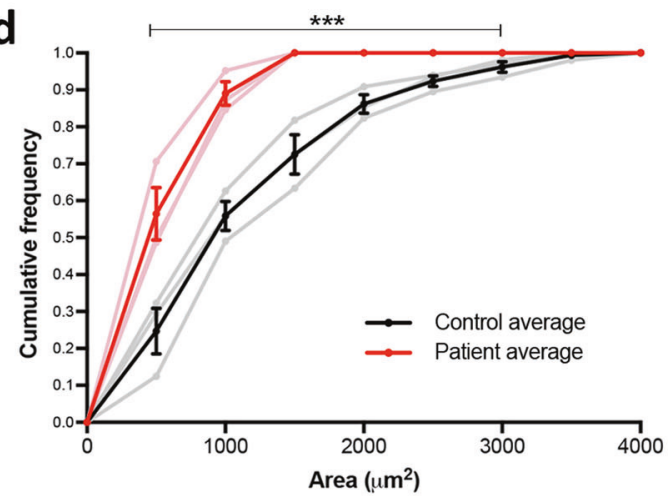

non-fluorescent resazurin to its fluorescent metabolite resorufin [28]. As evident throughout the entire 13-day time course of monitoring, OPCs derived from CSPG4 $4^{A 131 T}$ mutation carriers exhibited a significant decrease of cell viability (two-way repeated measures ANOVA, $P=8.9 \times 10^{-7}$; Fig. 3e).

OPCs are the exclusive precursor of oligodendrocytes [58]. Given the reduced viability of patient OPCs, we also 
Fig. 3 Aberrant NG2 subcellular localization, morphology, and viability of $C S P G 4^{A I 3 I T}$ patient OPCs. a, b Immunostaining for NG2 and calreticulin (CALR) reveals increased ER colocalization of NG2 in patient OPCs (scale bar $=10 \mu \mathrm{m}$ ). Quantification was performed in two independent experiments with three control and three patient OPC lines each $(t=5.08, P=0.007)$. c OPCs derived from mutation carriers exhibit an abnormally small morphology (scale bars $=10 \mu \mathrm{m}$ ). d Cumulative distribution of OPC area $\left(\mu \mathrm{m}^{2} ; n=361\right.$ control cells, $n$ $=217$ patient cells) demonstrates that OPCs derived from affected mutation carriers are significantly smaller than from their unaffected non-carrier siblings. Control and patient cell size distributions were compared by Kolmogorov-Smirnov test $\left(D=0.25, P=3.0 \times 10^{-8}\right)$. Dark lines show group mean \pm standard error. Gray and pink lines show the results from each of the individual control and patient subjects, respectively. e Fluorometric cell viability assay of OPCs derived from affected mutation carriers vs. their unaffected non-carrier siblings (two-way repeated measures ANOVA, $P=8.9 \times 10^{-7}$ ). f Representative z-stacked images of organotypic cerebral cortex slices of homozygous shiverer mice transplanted with OPCs derived from affected mutation carriers or their unaffected non-carrier siblings. Human nuclear antigen (hNA), green; MBP, red (scale bar $=100 \mu \mathrm{m})$. g MBP surface area normalized to $\mathrm{hNA}^{+}$cells per slice $(t=2.17$, $P=0.038)$

investigated whether their maturation to oligodendrocytes might also be impacted. Therefore, we performed ex vivo co-culture of control and patient OPCs with organotypic slices of cerebral cortex from myelin-deficient Shiverer mice that carry a homozygous mutation of $M b p$. The Shiverer myelination assay is a widely implemented method for assessing the myelination potential of OPCs, using culture conditions that promote OPCs differentiation to myelinating oligodendrocytes [30]. Consequently, MBP expression is entirely attributable to cells differentiated from the transplanted human OPCs, since Shiverer mice themselves lack Mbp. Consistent with their decreased viability, patient OPCs exhibited an impaired maturation to MBP-expressing oligodendrocytes $(t=2.17, P=0.038$; Fig. 3f, g).

\section{Transfection of OPCs with mutant isoforms of NG2}

To further investigate the causality of the $C S P G 4^{A 131 T}$ and CSPG $4^{V 901 G}$ mutations, we transfected either the wild-type (WT) or mutant isoforms into OPCs derived from healthy non-carrier siblings. Expression of CSPG4 $4^{A 131 T}$ recapitulated the abnormal retention in the endoplasmic reticulum, similarly as observed in patient OPCs (one-way ANOVA, WT vs. A131T: $P=5.2 \times 10^{-9}$; Fig. $\left.4 \mathrm{a}, \mathrm{b}\right)$. Moreover, OPCs expressing the $C S P G 4^{V 901 G}$ mutation also exhibited a significantly increased proportion of cells with colocalization to the endoplasmic reticulum compared to WT transfected OPCs (WT vs. V901G: $P=7.5 \times 10^{-6}$ ), albeit lower than observed for the $C S P G 4^{A 131 T}$ mutation. Notably, however, expression of the $C S P G 4^{V 901 G}$ mutation resulted in a distinct accumulation within putative intracellular vesicles that was evident in both OPCs (Fig. 4a, inset) and U373 glioblastoma cells (Supplementary Fig. 6), but not observed with expression of the CSPG4 $4^{A 131 T}$ mutation in either of these cell types. At present, although we have not yet succeeded in identifying the subcellular compartment to which these CSPG $4^{V 901 G}$-expressing intracellular vesicles belong, lysosomes have been excluded based on the lack of significant co-localization with lysosomal-associated membrane protein 1 (Supplementary Fig. 6).

Lastly, we sought to evaluate the causality of CSPG4 mutations for mediating the impaired cell viability observed for patient OPCs. Therefore, we performed transient transfections of either WT or mutant NG2 isoforms into OPCs derived from healthy non-carrier siblings and assessed cell survival after $48 \mathrm{~h}$ when plasmid expression was maximal. OPCs transfected with either CSPG $4^{A 131 T}$ or $C S P G 4^{V 901 G}$ exhibited a significant decrease in survival compared to CSPG4 ${ }^{\mathrm{WT}}$ (one-way ANOVA, WT vs. A131T: $P=0.006$; WT vs. V901G: $P=3.4 \times 10^{-4}$; Fig. 4c). Notably, we also attempted the same experiment with patient OPCs; however, patient OPCs consistently died following the transfection procedure, regardless of whether the transfection was performed using WT or mutant NG2, or empty vector, a finding that independently confirms their distinct vulnerability.

\section{Impaired white matter microstructure in CSPG4 ${ }^{A 131 T}$ mutation carriers}

Given the observed abnormalities of patient-derived OPCs, we reasoned that affected $C S P G 4^{A l 3 l T}$ mutation carriers might exhibit impairments of white matter integrity. Therefore, we performed brain MRI-based DTI in affected carrier and unaffected non-carrier siblings and compared them with 294 subjects from the general population Rotterdam Study cohort matched for age, gender, smoking behavior, and alcohol use (Fig. 5a). DTI images were analyzed for global and focal reductions in FA, the latter referred to as white matter potholes [59]. Affected carriers exhibited both a significantly higher number of white matter potholes $(P=$ $\left.2.2 \times 10^{-5}\right)$ and lower global FA $\left(P=8.2 \times 10^{-3}\right)$, compared to unaffected sibling and matched general population controls (potholes: $2.95 \pm 3.52$, 95\% CI 2.55-3.35; global FA: $0.415 \pm 0.015,95 \%$ CI $0.413-0.417$; Fig. $5 b, c)$.

\section{Discussion}

Our findings provide convergent genetic and functional neurobiological evidence that oligodendrocyte progenitor cell dysfunction might influence the pathophysiology of schizophrenia. We identified two different rare missense mutations in CSPG4 exhibiting familial segregation with schizophrenia. The discovery family $C S P G 4^{A 13 l T}$ mutation 
a
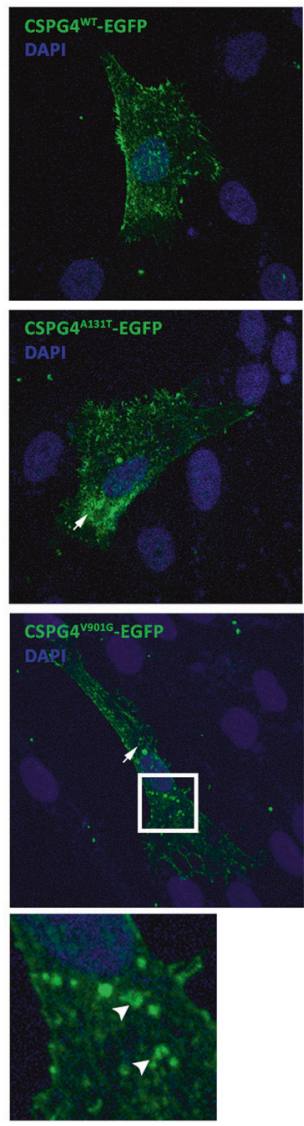
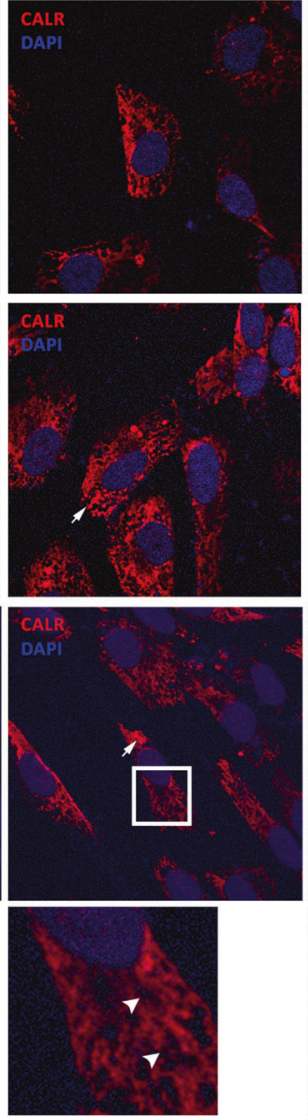
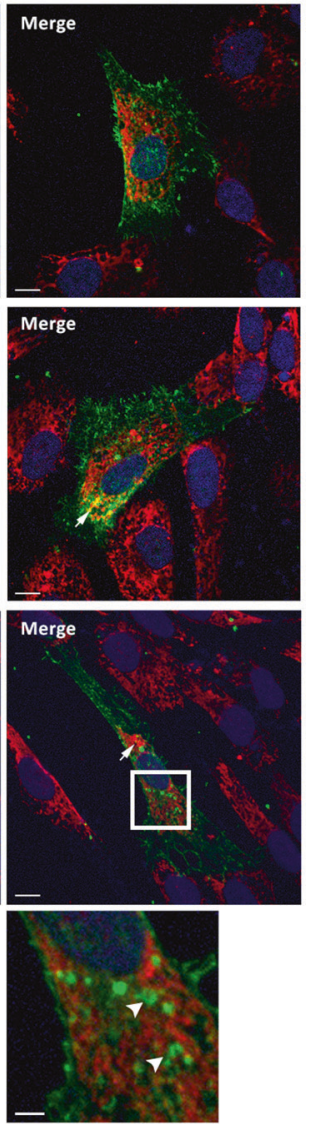

b

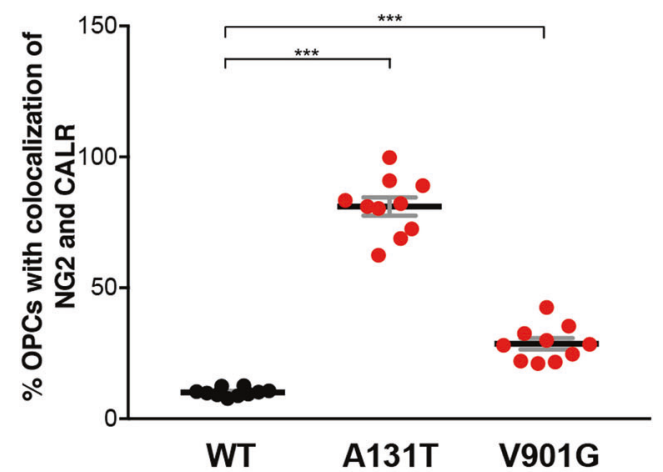

C

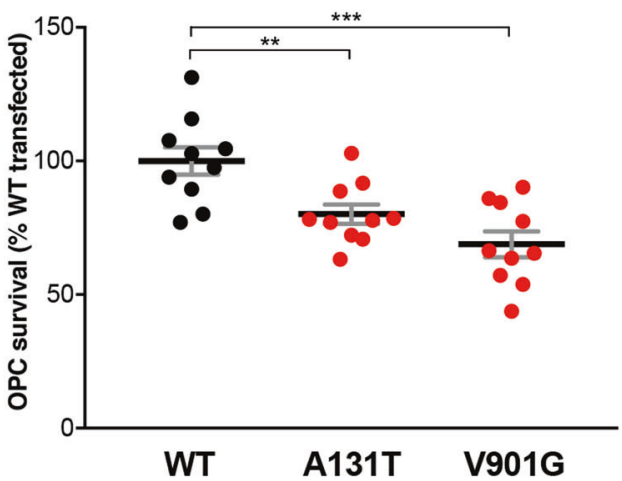

Fig. 4 Transfection of $C S P G 4^{A 131 T}$ and $C S P G 4^{V 901 G}$ mutations in healthy non-carrier sibling OPCs. Control OPCs were transiently transfected with plasmids expressing WT or mutant CSPG4 isoforms fused to EGFP. a Confocal images of transfected cells confirm a normal extracellular membrane-localization of CSPG4 ${ }^{\mathrm{WT}}$-EGFP. In contrast, a high proportion of OPCs transfected with CSPG4 ${ }^{A I 3 I T}$ EGFP exhibited colocalization with the endoplasmic reticulum marker calreticulin (CALR), reminiscent to patient OPCs with endogenous $C S P G 4^{A 131}$. OPCs expressing CSPG4 $4^{V 901 G}$-EGFP also revealed an increased proportion with CALR colocalization, but additionally demonstrated a distinctly abnormal targeting within putative intracellular vesicles that were negative for CALR (insets) (scale bars: main panels $=10 \mu \mathrm{m}$, insets $=3 \mu \mathrm{m}$ ). b Percentage of transfected OPCs with colocalization of CSPG4 isoforms with CALR (WT vs. A131T: $t=10.36, P=5.2 \times 10^{-9}$; WT vs. V901G: $t=6.20, P=7.5 \times 10^{-6}$ ). c Cell survival at $48 \mathrm{~h}$ after transfection of OPCs (WT vs. A131T: $t=3.11, P=0.006$; WT vs. V901G: $t=4.41, P=3.4 \times 10^{-4}$ )

segregation of two different rare CSPG4 missense variants with complete penetrance, none of these families was large enough on its own to achieve a genome-wide significant LOD score. Moreover, although the rare MAF of the CSPG4 $4^{A 131 T}$ mutation precluded case/control validation in the Swedish Schizophrenia Exome Sequencing Study, two of the three CSPG $4^{A 131 T}$ mutation carriers in the general population Rotterdam Study cohort had a clinically significant history of psychiatric illness that was unlikely due to chance. Furthermore, the CSPG $4^{V 901 G}$ mutation was present in the Swedish cohort with a nominally significant enrichment in cases vs. controls. Additional follow-up studies in larger cohorts will be required to definitively evaluate the association between schizophrenia and rare CSPG4 variants.

Genetic variation in $C S P G 4$ might confer a pleiotropic risk for mental illness since two of the CSPG $4^{A 13 l T}$ mutation carriers identified in the general population Rotterdam
Although we identified three independent families exhibiting 


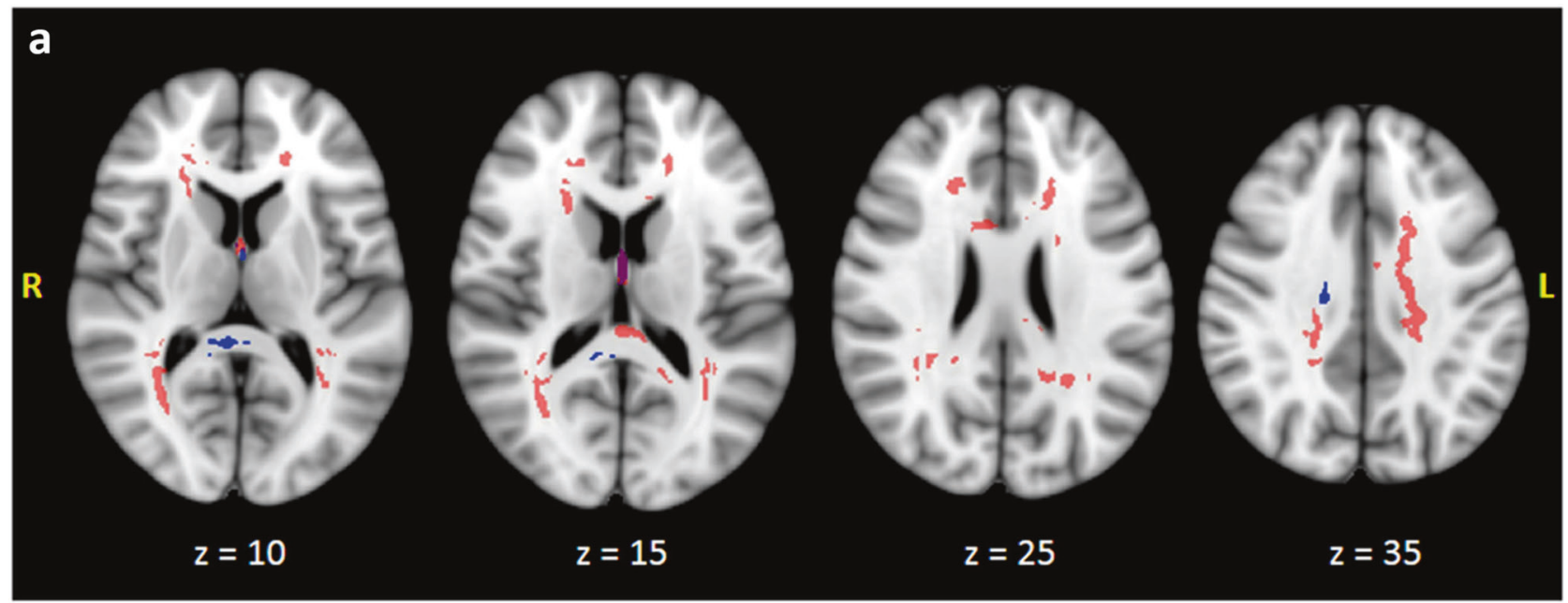

b

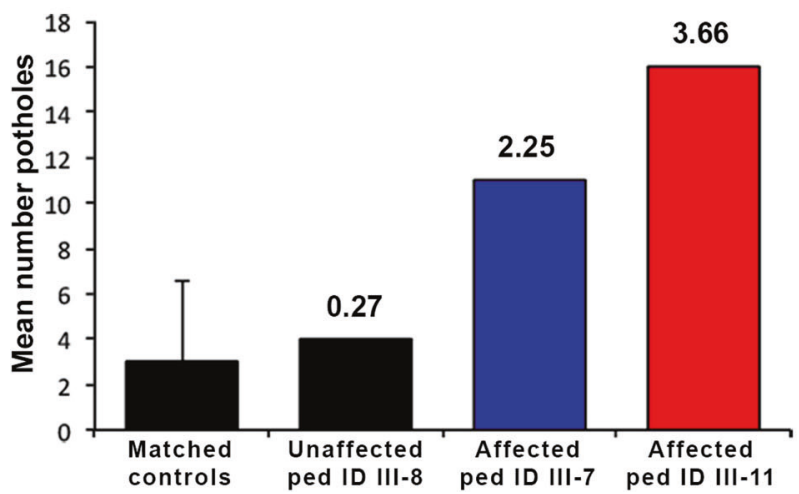

Fig. 5 White matter abnormalities in $C S P G 4^{A l 3 l T}$ patients. a White matter potholes found in the two affected family members are shown in red and blue, respectively. Purple regions define a spatial overlap of potholes in both patients. The $\mathrm{z}$-measures provide coordinates of the axial plane in MNI-space. b Mean number of potholes comparing the matched control population to the unaffected and two affected family

Study cohort had a clinically significant history of depression. Interestingly, a mouse model of OPC depletion was recently shown to exhibit depression-like behavior [60]. Such pleiotropic influences on mental health outcomes would be consistent with many of the previously identified genetic risk factors for schizophrenia [61, 62]. Furthermore, originally named as melanoma-associated chondroitin sulfate proteoglycan upon its cloning [63], NG2/CSPG4 has since been widely implicated in a wide variety of human cancers both as a diagnostic marker and a therapeutic target, including for glioblastoma and melanoma [64, 65].

The protein sequence surrounding the CSPG $4^{A 131 T}$ mutation is conserved only among higher-order primates, suggesting recent evolutionary pressure. In contrast, the $C S P G 4^{V 901 G}$ variant is predicted to be a disease-causing mutation by PolyPhen2 [66]. In non-primate vertebrates, the reference amino acid at position 131 is threonine,
C

Fractional Anisotropy

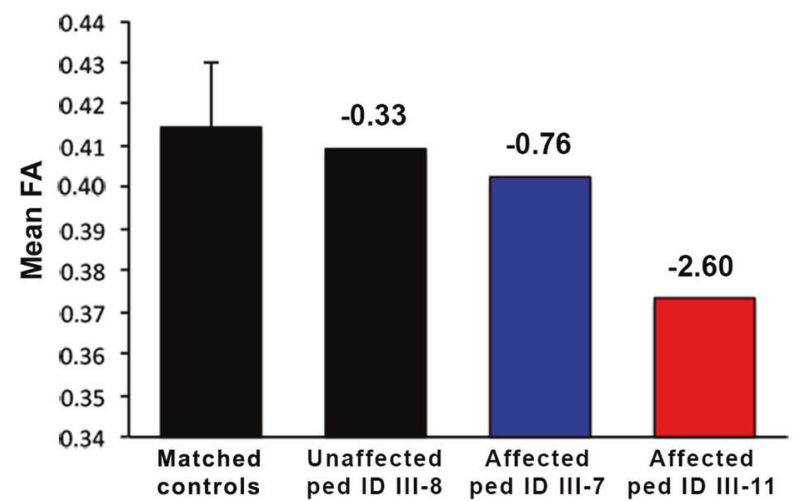

members. c Whole-brain white matter FA comparing the matched general population subjects to unaffected and affected family members. Error bars in $\mathbf{b}$ and $\mathbf{c}$ reflect the standard deviation of the matched general population subjects. The number above each bar reflects the individual $z$-score compared to the matched general population group

corresponding to the patient mutation $C S P G 4^{A 13 I T}$ and contributing to a benign PolyPhen score. Interestingly, this is analogous to the human $S N C A^{A 53 T}$ mutation, one of the most well-established mutations for autosomal dominant Parkinson's Disease [67]. In mice, the reference amino acid at $S C N A$ position 53 is a threonine. But notably, although transgenic expression in mice of the reference human SCNA sequence is benign, introduction of the human $S N C A^{A 53 T}$ mutation is highly pathogenic [67].

To investigate the cellular pathophysiology resulting from mutation of CSPG4, we derived iPSCs from affected and unaffected siblings of the discovery family. Detailed electrophysiological analysis of patient-derived neurons revealed an increase in input resistance and depolarized shift in AP threshold. Multiple previous studies have demonstrated primary functional neuronal mechanisms in human iPSC models of schizophrenia [3, 8, 11-13, 68]; 
however, the changes we observed are distinct from those previously identified. Therefore, future studies will be required to determine the extent to which these differences may have pathophysiological relevance, and potentially reflect an increased burden of common polygenic risk as recently observed in familial schizophrenia [69]. Importantly, however, our limited findings in neurons compared to OPCs cannot be considered to imply that neuronal dysfunction is not central to the pathophysiology of schizophrenia. Rather, these data suggest that although the symptoms of schizophrenia are ultimately manifest from neuronal dysfunction, the primary pathophysiological mechanism could be mediated by direct neuronal impairments and/or indirectly through nonneuronal cell types including OPCs, depending upon the nature of the etiological factors driving disease risk in a given individual.

OPCs receive extensive GABAergic input from surrounding neurons, which regulate their differentiation to myelinating oligodendrocytes [70-73]. However, it has remained less well understood whether OPCs directly modulate neuronal function independent of myelination. Notably, two recent studies have suggested novel candidate mechanisms by which OPC dysfunction might directly regulate neuronal function, including activity-dependent ecto-domain cleavage of NG2 [54] and local buffering of extracellular potassium [74].

Regarding the identified CSPG4 mutations demonstrating familial segregation with schizophrenia, alterations in myelination are a parsimonious candidate mechanism given the convergent findings of genetic mutations in the OPC marker protein CSPG4, functional impairments of iPSCderived OPCs, and in vivo DTI-based structural brain imaging. Moreover, the abnormal subcellular localization of NG2 in OPCs expressing the CSPG4 $4^{A 131 T}$ or CSPG $4^{V 901 G}$ isoforms is consistent with the high susceptibility of cells of the oligodendrocyte lineage to disruptions in the secretory pathway, as their maturation requires a substantial upregulation of membrane protein expression [75]. Interestingly, earlier work has shown a precedent for altered subcellular localization of NG2, in which mutation of Ser $^{999}$ results in an abrogation of chondroitin sulfate side chain modification and altered subcellular localization [55].

Taken together, these findings are highly consistent with the growing body of evidence implicating white matter integrity in schizophrenia neuropathology [59, 76-79]. Indeed, recent findings demonstrating myelination of parvalbumin-positive GABAergic interneurons-arguably the most well-established neuronal cell type implicated in the pathophysiology of schizophrenia [80, 81] —raises the intriguing possibility that schizophrenia might result from neurodevelopmental alterations of PV interneuron myelination [82].
In summary, our findings support the validity of familybased genetics and iPSC modeling to unravel the underlying mechanisms of complex, heterogeneous psychiatric diseases, and provide evidence in support of oligodendrocyte precursor cell dysfunction as a novel candidate mechanism of schizophrenia.

Acknowledgements We wish to thank Roel Ophoff for facilitating the contribution of the GROUP/Utrecht Study samples and discussions regarding the genetic analyses, Gerard Borst for discussions regarding the electrophysiological analyses, and Siska Verploegh for her assistance in sample collection. This project was partially funded by the Erasmus MC-University Medical Centre Rotterdam, the Netherlands Organization for Scientific Research (NWO) and Netherlands Organisation for Health Research and Development (ZonMW) to SAK, VB, YE, and JG, the NeuroBasic-PharmaPhenomics consortium to SAK and YE, Stichting ParkinsonFonds (The Netherlands) to VB, Netherlands Institute for Regenerative Medicine (NIRM) and European Research Council (Consolidator Grant) to JG. The authors would like to thank the NHLBI GO Exome Sequencing Project and its ongoing studies which produced and provided exome variant calls for comparison: the Lung GO Sequencing Project (HL-102923), the WHI Sequencing Project (HL-102924), the Broad GO Sequencing Project (HL-102925), the Seattle GO Sequencing Project (HL-102926), and the Heart GO Sequencing Project (HL-103010). This study makes use of data generated by the Genome of the Netherlands Project. A full list of the investigators is available from www.nlgenome.nl. Funding for the project was provided by the Netherlands Organization for Scientific Research under award number 184021007, dated 9 July 2009 and made available as a Rainbow Project of the Biobanking and Biomolecular Research Infrastructure Netherlands (BBMRI-NL). The sequencing was carried out in collaboration with the Beijing Institute for Genomics (BGI). The generation and management of genomics data for the Rotterdam Study were supported by the Netherlands Organisation of Scientific Research Investments (nr. 175. 010.2005.011, 911-03-012) and the Netherlands Genomics Initiative (NGI) project nr. 050-060-810 (Netherlands Consortium for Healthy Ageing; NCHA). We thank the members of the Human Genomics Facility (HuGeF) and the ERGO support team for their help in sampling the data and in creating the database. The Rotterdam Study is funded by Erasmus Medical Centre and Erasmus University, Rotterdam, Netherlands Organisation for Health Research and Development (ZonMw), the Research Institute for Diseases in the Elderly (RIDE), the Ministry of Education, Culture and Science, the Ministry for Health, Welfare and Sports, the European Commission (DG XII), and the Municipality of Rotterdam. The authors are grateful to the study participants, the staff from the Rotterdam Study and the participating general practitioners and pharmacists. NIMH Study 13-data used in this research report were collected by the International Neuro-Genetics Association of Spanish America and the United States (INGASU), and funded by a collaborative NIMH grant (Genetics of Schizophrenia in Latino Populations) to Dr. Michael Escamilla (University of Texas Health Science Center at San Antonio) (MH60881) and to Dr. Ricardo Mendoza (University of California at Los Angeles-Harbor) (MH60875). Additional principal investigators who participated in these grants were Dr. Henriette Raventos (University of Costa Rica, San Jose, Costa Rica), Dr. Alfonso Ontiveros (Instituto de Informacion de Investigacion en Salud Mental, Monterrey, Mexico), Dr. Humberto Nicolini (Medical and Family Research Group, Carracci S.C., Mexico City, Mexico), Dr. Rodrigo Munoz (Family Health Centers of San Diego, California), and Dr. Alvaro Jerez (Centro Internacional de Trastornos Afectivos y de la Conducta Adictiva-CITACA, Guatemala). Additional investigators from the University of Texas Health Science Center at San Antonio included Dr. Albana Dassori and Dr. 
Rolando Medina. Swedish Schizophrenia Exome Sequencing project: Data obtained from dbGaP at http://www.ncbi.nlm.nih.gov/gap through dbGaP accession number (20 September 2015, accession phs000473.v1.p1). Samples used for data analysis were provided by the Swedish Cohort Collection supported by the NIMH grant R01MH077139, the Sylvan C. Herman Foundation, the Stanley Medical Research Institute, and The Swedish Research Council (grants 2009-4959 and 2011-4659). Support for the exome sequencing was provided by the NIMH Grand Opportunity grant RCMH089905, the Sylvan C. Herman Foundation, a grant from the Stanley Medical Research Institute and multiple gifts to the Stanley Center for Psychiatric Research at the Broad Institute of MIT and Harvard. The authors would like to thank the Exome Aggregation Consortium and the groups that provided exome variant data for comparison. A full list of contributing groups can be found at https://exac.broadinstitute. org/about.

\section{Compliance with ethical standards}

Conflict of interest The authors declare that they have no conflict of interest.

Open Access This article is licensed under a Creative Commons Attribution 4.0 International License, which permits use, sharing, adaptation, distribution and reproduction in any medium or format, as long as you give appropriate credit to the original author(s) and the source, provide a link to the Creative Commons license, and indicate if changes were made. The images or other third party material in this article are included in the article's Creative Commons license, unless indicated otherwise in a credit line to the material. If material is not included in the article's Creative Commons license and your intended use is not permitted by statutory regulation or exceeds the permitted use, you will need to obtain permission directly from the copyright holder. To view a copy of this license, visit http://creativecommons. org/licenses/by/4.0/.

\section{References}

1. Owen MJ, Sawa A, Mortensen PB. Schizophrenia. Lancet 2016;6736:1-12.

2. Polderman TJC, Benyamin B, de Leeuw CA, Sullivan PF, van Bochoven A, Visscher PM, et al. Meta-analysis of the heritability of human traits based on fifty years of twin studies. Nat Genet 2015;47:702-9.

3. Ripke S, Neale BM, Corvin A, Walters JTR, Farh K-H, Holmans $\mathrm{PA}$, et al. Biological insights from 108 schizophrenia-associated genetic loci. Nature. 2014;511:421-7.

4. Sullivan PF, Daly MJ, O'Donovan M. Genetic architectures of psychiatric disorders: the emerging picture and its implications. Nat Rev Genet. 2012;13:537-51.

5. Rees E, Walters JTR, Georgieva L, Isles AR, Chambert KD, Richards AL, et al. Analysis of copy number variations at 15 schizophrenia-associated loci. Br J Psychiatry. 2014;204:108-14.

6. Marshall C, Howrigan D, Merico D, Thiruvahindrapuram B, Wu W, Greer D, et al. A contribution of novel CNVs to schizophrenia from a genome-wide study of 41,321 subjects. Cold Spring Harb Labs J. 2016;49:27-35.

7. Szatkiewicz JP, O'Dushlaine C, Chen G, Chambert K, Moran JL, Neale BM, et al. Copy number variation in schizophrenia in Sweden. Mol Psychiatry. 2014;19:762-73.

8. Purcell SM, Moran JL, Fromer M, Ruderfer D, Solovieff N, Roussos $\mathrm{P}$, et al. A polygenic burden of rare disruptive mutations in schizophrenia. Nature. 2014;506:185-90.
9. Takata A, Ionita-Laza I, Gogos JA, Xu B, Karayiorgou M. De novo synonymous mutations in regulatory elements contribute to the genetic etiology of autism and schizophrenia. Neuron. 2016;89:940-7.

10. Xu B, Ionita-Laza I, Roos JL, Boone B, Woodrick S, Sun Y, et al. De novo gene mutations highlight patterns of genetic and neural complexity in schizophrenia. Nat Genet. 2012;44: 1365-9.

11. Fromer M, Pocklington AJ, Kavanagh DH, Williams HJ, Dwyer $\mathrm{S}$, Gormley $\mathrm{P}$, et al. De novo mutations in schizophrenia implicate synaptic networks. Nature. 2014;506:179-84.

12. Brennand KJ, Simone A, Jou J, Gelboin-Burkhart C, Tran N, Sangar S, et al. Modelling schizophrenia using human induced pluripotent stem cells. Nature. 2011;473:221-5.

13. Sekar A, Bialas AR, de Rivera H, Davis A, Hammond TR, Kamitaki N, et al. Schizophrenia risk from complex variation of complement component 4. Nature. 2016;530:177-83.

14. Nave K-A, Ehrenreich H. Myelination and oligodendrocyte functions in psychiatric diseases. JAMA Psychiatry. 2014;71: $582-4$.

15. Goudriaan A, de Leeuw C, Ripke S, Hultman CM, Sklar P, Sullivan $\mathrm{PF}$, et al. Specific glial functions contribute to schizophrenia susceptibility. Schizophr Bull. 2014;40:925-35.

16. Fields RD. White matter in learning, cognition and psychiatric disorders. Trends Neurosci. 2008;31:361-70.

17. Paus T, Keshavan M, Giedd JN. Why do many psychiatric disorders emerge during adolescence? Nat Rev Neurosci. 2008;9:947-57.

18. Terwisscha Van Scheltinga AF, Bakker SC, Van Haren NEM, Derks EM, Buizer-Voskamp JE, Boos HBM, et al. Genetic schizophrenia risk variants jointly modulate total brain and white matter volume. Biol Psychiatry. 2013;73:525-31.

19. van den Heuvel MP, Sporns O, Collin G, Scheewe T, Mandl $\mathrm{RCW}$, Cahn W, et al. Abnormal rich club organization and functional brain dynamics in schizophrenia. JAMA Psychiatry. 2013;70:783-92.

20. Voineskos AN, Lobaugh NJ, Bouix S, Rajji TK, Miranda D, Kennedy JL, et al. Diffusion tensor tractography findings in schizophrenia across the adult lifespan. Brain. 2010;133: 1494-504.

21. Chavarria-Siles I, White T, de Leeuw C, Goudriaan A, Lips E, Ehrlich S, et al. Myelination-related genes are associated with decreased white matter integrity in schizophrenia. Eur J Hum Genet. 2016;24:381-6.

22. Duncan LE, Holmans PA, Lee PH, O'Dushlaine CT, Kirby AW, Smoller JW, et al. Pathway analyses implicate glial cells in schizophrenia. PLoS ONE. 2014;9:e89441.

23. Chang YS, Owen JP, Pojman NJ, Thieu T, Bukshpun P, Wakahiro MLJ, et al. Reciprocal white matter alterations due to $16 \mathrm{p} 11.2$ chromosomal deletions versus duplications. Hum Brain Mapp. 2016;37:2833-48.

24. Owen JP, Chang YS, Pojman NJ, Bukshpun P, Wakahiro MLJ, Marco EJ, et al. Aberrant white matter microstructure in children with 16p11.2 deletions. J Neurosci. 2014;34:6214-23.

25. Gunhanlar N, Shpak G, van der Kroeg M, Gouty-Colomer LA, Munshi ST, Lendemeijer B et al. A simplified protocol for differentiation of electrophysiologically mature neuronal networks from human induced pluripotent stem cells. Mol Psychiatry. 2017; doi: $10.1038 / \mathrm{mp} .2017 .56$.

26. Monaco MCG, Maric D, Bandeian A, Leibovitch E, Yang W, Major EO. Progenitor-derived oligodendrocyte culture system from human fetal brain. J Vis Exp. 2012;70:4274.

27. Huang GN, Zeng W, Kim JY, Yuan JP, Han L, Muallem S, et al. STIM1 carboxyl-terminus activates native SOC, I(crac) and TRPC1 channels. Nat Cell Biol. 2006;8:1003-10. 
28. Back SA, Khan R, Gan X, Rosenberg PA, Volpe JJ. A new Alamar Blue viability assay to rapidly quantify oligodendrocyte death. J Neurosci Methods. 1999;91:47-54.

29. Stoppini L, Buchs PA, Muller D. A simple method for organotypic cultures of nervous tissue. $\mathrm{J}$ Neurosci Methods. 1991;37:173-82.

30. Najm FJ, Lager AM, Zaremba A, Wyatt K, Caprariello AV, Factor DC, et al. Transcription factor-mediated reprogramming of fibroblasts to expandable, myelinogenic oligodendrocyte progenitor cells. Nat Biotechnol. 2013;31:426-33.

31. Ikram MA, van der Lugt A, Niessen WJ, Krestin GP, Koudstaal PJ, Hofman A, et al. The Rotterdam Scan Study: design and update up to 2012. Eur J Epidemiol. 2011;26:811-24.

32. White T, Schmidt M, Karatekin C. White matter 'potholes' in early-onset schizophrenia: a new approach to evaluate white matter microstructure using diffusion tensor imaging. Psychiatry Res. 2009;174:110-5.

33. Exome Aggregation Consortium, Lek M, Karczewski K, Minikel E, Samocha K, Banks E, et al. Analysis of protein-coding genetic variation in 60,706 humans. Cold Spring Harb Labs J. 2015;536:285-91.

34. Exome Variant Server, NHLBI GO Exome Sequencing Project (ESP), Seattle, WA. 2013. https://evs.gs.washington.edu/EVS/

35. Abecasis GR, Altshuler D, Auton A, Brooks LD, Durbin RM, Gibbs RA, et al. A map of human genome variation from population-scale sequencing. Nature. 2010;467:1061-73.

36. Consortium TG of the N. Whole-genome sequence variation, population structure and demographic history of the Dutch population. Nat Genet. 2014; 46:818-25.

37. Hofman A, Murad SD, Van Duijn CM, Franco OH, Goedegebure A, Arfan Ikram M, et al. The Rotterdam Study: 2014 objectives and design update. Eur J Epidemiol. 2013;28:889-926.

38. Dekker J, Peen J, Gardien R, de Jonghe F, Wijdenes W. Urbanisation and psychiatric admission rates in The Netherlands. Int $\mathbf{J}$ Soc Psychiatry. 1997;43:235-46.

39. Wierdsma AI, Van Marle PD, Mulder CL. Aantallen en patronen van inbewaringstellingen en rechterlijke machtigingen in Maastricht, Groningen en Rotterdam: Bijdrage van stedelijke problematiek aan regionale verschillen in Bopz-maatregelen. Tijdschr Psychiatr. 2010;52:143-53.

40. Escamilla M, Hare E, Dassori AM, Peralta JM, Ontiveros A, Nicolini $\mathrm{H}$, et al. A schizophrenia gene locus on chromosome $17 q 21$ in a new set of families of Mexican and Central American ancestry: evidence from the NIMH genetics of schizophrenia in latino populations study. Am J Psychiatry. 2009;166:442-9.

41. Vieland VJ, Walters KA, Azaro M, Brzustowicz LM, Lehner T. The value of regenotyping older linkage data sets with denser marker panels. Hum Hered. 2014;78:9-16.

42. Timpl R, Tisi D, Talts JF, Andac Z, Sasaki T, Hohenester E. Structure and function of laminin LG modules. Matrix Biol. 2000;19:309-17.

43. Xu B, Roos JL, Dexheimer P, Boone B, Plummer B, Levy S, et al. Exome sequencing supports a de novo mutational paradigm for schizophrenia. Nat Genet. 2011;43:1-6.

44. Rujescu D, Ingason A, Cichon S, Pietiläinen OPH, Barnes MR, Toulopoulou T, et al. Disruption of the neurexin 1 gene is associated with schizophrenia. Hum Mol Genet. 2009;18:988-96.

45. Kirov G, Gumus D, Chen W, Norton N, Georgieva L, Sari M, et al. Comparative genome hybridization suggests a role for NRXN1 and APBA2 in schizophrenia. Hum Mol Genet. 2008;17:458-65.

46. Kelley LA, Sternberg MJE. Protein structure prediction on the web: a case study using the Phyre server. Nat Protoc. 2009;4:363-71.

47. Zhang Y. I-TASSER server for protein 3D structure prediction. BMC Bioinforma. 2008;9:40.
48. Miller MT, Mileni M, Comoletti D, Stevens RC, Harel M, Taylor $\mathrm{P}$. The crystal structure of the $\alpha$-neurexin- 1 extracellular region reveals a hinge point for mediating synaptic adhesion and function. Structure. 2011;19:767-78.

49. Chen F, Venugopal V, Murray B, Rudenko G. The structure of neurexin $1 \alpha$ reveals features promoting a role as synaptic organizer. Structure. 2011;19:779-89.

50. Holm L, Rosenström P. Dali server: conservation mapping in 3D. Nucleic Acids Res. 2010;38:W545-9.

51. Burg MA, Nishiyama A, Stallcup WB. A central segment of the NG2 proteoglycan is critical for the ability of glioma cells to bind and migrate toward type VI collagen. Exp Cell Res. 1997;235:254-64.

52. Tillet E, Gential B, Garrone R, Stallcup WB. NG2 proteoglycan mediates beta1 integrin-independent cell adhesion and spreading on collagen VI. J Cell Biochem. 2002;86:726-36.

53. Nishiyama A, Lin XH, Stallcup WB. Generation of truncated forms of the NG2 proteoglycan by cell surface proteolysis. Mol Biol Cell. 1995;6:1819-32.

54. Sakry D, Neitz A, Singh J, Frischknecht R, Marongiu D, Binamé $\mathrm{F}$, et al. Oligodendrocyte precursor cells modulate the neuronal network by activity-dependent ectodomain cleavage of glial NG2. PLoS Biol. 2014;12:e1001993.

55. Stallcup WB, Dahlin-Huppe K. Chondroitin sulfate and cytoplasmic domain-dependent membrane targeting of the NG2 proteoglycan promotes retraction fiber formation and cell polarization. J Cell Sci. 2001;114:2315-25.

56. Obacz J, Avril T, Le Reste P-J, Urra H, Quillien V, Hetz C, et al. Endoplasmic reticulum proteostasis in glioblastoma-from molecular mechanisms to therapeutic perspectives. Sci Signal. 2017; 10:eaal2323.

57. Clayton BLL, Popko B. Endoplasmic reticulum stress and the unfolded protein response in disorders of myelinating glia. Brain Res. 2016;1648:594-602.

58. Bergles DE, Richardson WD. Oligodendrocyte development and plasticity. Cold Spring Harb Perspect Biol. 2016;8:a020453.

59. White T, Ehrlich S, Ho B, Manoach DS, Caprihan A, Schulz SC, et al. Spatial characteristics of white matter abnormalities in schizophrenia. Schizophr Bull. 2013;39:1077-86.

60. Birey F, Kloc M, Chavali M, Hussein I, Wilson M, Christoffel DJ, et al. Genetic and stress-induced loss of NG2 glia triggers emergence of depressive-like behaviors through reduced secretion of FGF2. Neuron. 2015;88:941-56.

61. Lee SH, Ripke S, Neale BM, Faraone SV, Purcell SM, Perlis RH, et al. Genetic relationship between five psychiatric disorders estimated from genome-wide SNPs. Nat Genet. 2013;45:984-94.

62. O'Donovan MC, Owen MJ. The implications of the shared genetics of psychiatric disorders. Nat Med. 2016;22:1214-9.

63. Pluschke G, Vanek M, Evans A, Dittmar T, Schmid P, Itin P, et al. Molecular cloning of a human melanoma-associated chondroitin sulfate proteoglycan. Proc Natl Acad Sci USA. 1996;93:9710-5.

64. Riccardo F, Iussich S, Maniscalco L, Lorda Mayayo S, La Rosa G, Arigoni M, et al. CSPG4-specific immunity and survival prolongation in dogs with oral malignant melanoma immunized with human CSPG4 DNA. Clin Cancer Res. 2014;20: 3753-62.

65. Geldres C, Savoldo B, Hoyos V, Caruana I, Zhang M, Yvon E, et al. $\mathrm{T}$ lymphocytes redirected against the chondroitin sulfate proteoglycan-4 control the growth of multiple solid tumors both in vitro and in vivo. Clin Cancer Res. 2014;20:962-71.

66. Adzhubei IA, Schmidt S, Peshkin L, Ramensky VE, Gerasimova A, Bork P, et al. A method and server for predicting damaging missense mutations. Nat Methods. 2010;7:248-9. 
67. Lee MK, Stirling W, Xu Y, Xu X, Qui D, Mandir AS, et al. Human -synuclein-harboring familial Parkinson's disease-linked Ala-53 $\rightarrow$ Thr mutation causes neurodegenerative disease with -synuclein aggregation in transgenic mice. Proc Natl Acad Sci USA. 2002;99:8968-73.

68. Wen Z, Nguyen HN, Guo Z, Lalli MA, Wang X, Su Y, et al. Synaptic dysregulation in a human iPS cell model of mental disorders. Nature. 2014;515:414-8.

69. Bigdeli TB, Ripke S, Bacanu S-A, Lee SH, Wray NR, Gejman PV, et al. Genome-wide association study reveals greater polygenic loading for schizophrenia in cases with a family history of illness. Am J Med Genet B Neuropsychiatr Genet. 2016;171:276-89.

70. Lin S, Bergles DE. Synaptic signaling between GABAergic interneurons and oligodendrocyte precursor cells in the hippocampus. Nat Neurosci. 2004;7:24-32.

71. Orduz D, Maldonado PP, Balia M, Vélez-Fort M, de Sars V, Yanagawa $\mathrm{Y}$ et al. Interneurons and oligodendrocyte progenitors form a structured synaptic network in the developing neocortex. eLife 2015;4:e06953

72. Zonouzi M, Scafidi J, Li P, McEllin B, Edwards J, Dupree JL, et al. GABAergic regulation of cerebellar NG2 cell development is altered in perinatal white matter injury. Nat Neurosci. 2015;18:674-82.

73. Balia M, Vélez-Fort M, Passlick S, Schäfer C, Audinat E, Steinhäuser $\mathrm{C}$, et al. Postnatal down-regulation of the GABAA receptor $\gamma 2$ subunit in neocortical NG2 cells accompanies synaptic-toextrasynaptic switch in the GABAergic transmission mode. Cereb Cortex. 2015;25:1114-23.
74. Maldonado PP, Vélez-Fort M, Levavasseur F, Angulo MC. Oligodendrocyte precursor cells are accurate sensors of local $\mathrm{K}+$ in mature gray matter. J Neurosci. 2013;33: 2432-42.

75. Lin W, Popko B. Endoplasmic reticulum stress in disorders of myelinating cells. Nat Neurosci. 2009;12:379-85.

76. Zalesky A, Fornito A, Seal ML, Cocchi L, Westin CF, Bullmore ET, et al. Disrupted axonal fiber connectivity in schizophrenia. Biol Psychiatry. 2011;69:80-9.

77. Davis KL, Stewart DG, Friedman JI, Buchsbaum M, Harvey PD, Hof PR, et al. White matter changes in schizophrenia: evidence for myelin-related dysfunction. Arch Gen Psychiatry. 2003;60: 443-56.

78. Andreasen NC, Nopoulos P, Magnotta V, Pierson R, Ziebell S, Ho BC. Progressive brain change in schizophrenia: a prospective longitudinal study of first-episode schizophrenia. Biol Psychiatry. 2011;70:672-9.

79. Windrem MS, Osipovitch M, Liu Z, Bates J, Chandler-Militello D, Zou L, et al. Human iPSC glial mouse chimeras reveal glial contributions to schizophrenia. Cell Stem Cell. 2017;21:195-208.

80. Gonzalez-Burgos G, Cho RY, Lewis DA. Alterations in cortical network oscillations and parvalbumin neurons in schizophrenia. Biol Psychiatry. 2015;77:1031-40.

81. Marín O. Developmental timing and critical windows for the treatment of psychiatric disorders. Nat Med. 2016;22:1229-38.

82. Stedehouder J, Kushner SA. Myelination of parvalbumin interneurons: a parsimonious locus of pathophysiological convergence in schizophrenia. Mol Psychiatry. 2017;22:4-12.

\section{Affiliations}

Femke M. de Vrij ${ }^{1} \cdot$ Christian G. Bouwkamp ${ }^{1,2} \cdot$ Nilhan Gunhanlar $^{1} \cdot$ Guy Shpak $^{1} \cdot$ Bas Lendemeijer $^{1}$. Maarouf Baghdadi ${ }^{1}$. Shreekara Gopalakrishna $\mathbb{1}^{1} \cdot$ Mehrnaz Ghazvini $^{3} \cdot$ Tracy M. $\mathrm{Li}^{3} \cdot$ Marialuisa Quadri ${ }^{2}$. Simone Olgiati ${ }^{2} \cdot$ Guido J. Breedveld $^{2} \cdot$ Michiel Coesmans $^{1,4} \cdot$ Edwin Mientjes $^{5,6} \cdot$ Ton de Wit $^{2} \cdot$ Frans W. Verheijen $^{2}$. H. Berna Beverloo ${ }^{2}$ Dan Cohen ${ }^{7} \cdot$ Rob M. Kok $^{8} \cdot$ P. Roberto Bakker ${ }^{9,10} \cdot$ Aviva Nijburg $^{8}$ - Annet T. Spijker ${ }^{8}$. P.M. Judith Haffmans ${ }^{11}$ • Erik Hoencamp ${ }^{8,12}$ • Veerle Bergink ${ }^{1}$ - GROUP Study Consortium • Jacob A. Vorstman ${ }^{13,14,15} \cdot$ Timothy $\mathrm{Wu}^{16} \cdot$ Loes M. Olde Loohuis $\mathbb{D}^{16} \cdot$ Najaf Amin $^{17} \cdot$ Carolyn D. Langen ${ }^{18,19,20}$. Albert Hofman ${ }^{17}$ - Witte J. Hoogendijk ${ }^{1}$. Cornelia M. van Duijn ${ }^{17} \cdot$ M. Arfan Ikram (i) ${ }^{17,18,21} \cdot$ Meike W. Vernooij ${ }^{17,18}$. Henning Tiemeier $\mathbb{D}^{1,17,19} \cdot$ André G. Uitterlinden ${ }^{17,22} \cdot$ Ype Elgersma $\mathbb{D}^{5,6} \cdot$ Ben Distel $^{23} \cdot$ Joost Gribnau $^{3}$. Tonya White ${ }^{19} \cdot$ Vincenzo Bonifati ${ }^{2}$ Steven A. Kushner (1) 1

1 Department of Psychiatry, Erasmus Medical Center, Rotterdam, The Netherlands

2 Department of Clinical Genetics, Erasmus Medical Center, Rotterdam, The Netherlands

3 Department of Developmental Biology, and Erasmus MC iPS Facility, Erasmus Medical Center, Rotterdam, The Netherlands

4 Delta Psychiatric Center, Poortugaal, The Netherlands

5 Department of Neuroscience, Erasmus Medical Center, Rotterdam, The Netherlands

6 ENCORE Expertise Center for Neurodevelopmental Disorders, Erasmus Medical Center, Rotterdam, The Netherlands

7 Mental Health Care Organization North-Holland North, Heerhugowaard, The Netherlands

8 Parnassia Psychiatric Institute, The Hague, The Netherlands
9 Department of Psychiatry and Psychology, School of Mental Health and Neuroscience, Maastricht University Medical Center, Maastricht, The Netherlands

10 Psychiatric Center GGZ Centraal, Amersfoort, The Netherlands

11 Faculty of Social and Behavioral Sciences Clinical, Health and Neuro Psychology, Department of Affective Disorders, PsyQ, Leiden University, Leiden, The Netherlands

12 Institute of Psychology, Leiden University, Leiden, The Netherlands

13 Department of Psychiatry, Brain Center Rudolf Magnus, University Medical Center Utrecht, Utrecht, The Netherlands

14 Department of Psychiatry, The Hospital for Sick Children and University of Toronto, Toronto, Ontario, Canada

15 Program in Genetics and Genome Biology, Research Institute, The Hospital for Sick Children, Toronto, Ontario, Canada 
16 Center for Neurobehavioral Genetics, Semel Institute for Neuroscience and Human Behavior, University of California, Los Angeles, CA, USA

17 Department of Epidemiology, Erasmus Medical Center, Rotterdam, The Netherlands

18 Department of Radiology, Erasmus Medical Center, Rotterdam, The Netherlands

19 Department of Medical Informatics, Erasmus Medical Center, Rotterdam, The Netherlands
20 Biomedical Imaging Group Rotterdam, Departments of Radiology \& Medical Informatics, Erasmus Medical Center, Rotterdam, The Netherlands

21 Department of Neurology, Erasmus Medical Center, Rotterdam, The Netherlands

22 Department of Internal Medicine, Erasmus Medical Center, Rotterdam, The Netherlands

23 Department of Medical Biochemistry, Academic Medical Centre, Amsterdam, The Netherlands 